\title{
Image Encryption in Transform Domain using Circular Shift
}

\author{
Tanuja Sarode, PhD \\ Thadomal Shahani \\ Engineering College \\ Affiliated to Mumbai \\ University, India
}

\author{
Devishree Pillai \\ Thadomal Shahani \\ Engineering College \\ Affiliated to Mumbai \\ University, India
}

\author{
Shalmalee Pokale \\ Thadomal Shahani \\ Engineering College \\ Affiliated to Mumbai \\ University, India
}

\author{
Tejashri Prabhu \\ Thadomal Shahani \\ Engineering College \\ Affiliated to Mumbai \\ University, India
}

\begin{abstract}
With the advent of cloud storage and increase in transmission images through internet, images are more vulnerable to attacks of various hackers. A lot of private images are leaked from the cloud. Information security has become a major concern. Also, as the use of digital techniques for transmitting and storing images are increasing, it becomes an important issue that how to protect the confidentiality, integrity and authenticity of images. Encrypting the images is one way of dealing with this threat. Image encryption techniques try to convert original image to another image which is hard to decipher so that the image is kept confidential between users, in other words, it is essential that nobody will get to know the content without a key for decryption. This paper proposes a new approach to image encryption using circular shift in transform domain.
\end{abstract}

\section{General Terms}

Image encryption, Security

\section{Keywords}

Circular shift, Discrete Cosine Transform

\section{INTRODUCTION}

Encryption is a process of converting the message into a coded format (cipher) which ensures that the message is unintelligible to anyone without means to decipher it [1]. Decryption is converting the cipher back to the original message so that it is readable [1]. Encrypting data provides confidentiality, integrity and authentication [2]. Traditional text encryptions cannot be used for encrypting images as they are much larger in size which causes overhead and is a costly process [3]. The vicinal pixels are highly correlated in images and hence text encryptions fail to conceal the entire Image; also the images have patterns and repetitive backgrounds which are not taken into account by the text encryptions and this increases redundancy [3]. Also complete accuracy is not needed in image encryption unlike text encryption. Hence conventional encryption algorithms like Data Encryption Standard (DES), Advanced Encryption Standard (AES), Blowfish, and International Data Encryption Standard (IDEA) are not suitable for image encryption, hence encryption algorithms made exclusively for images are needed [3]. This paper presents image encryption in transform domain; in which a series of row or columnar circular shifts are applied to a DCT transformed image and the image is decrypted back by undoing those circular shifts.

\section{SYSTEM DESCRIPTION}

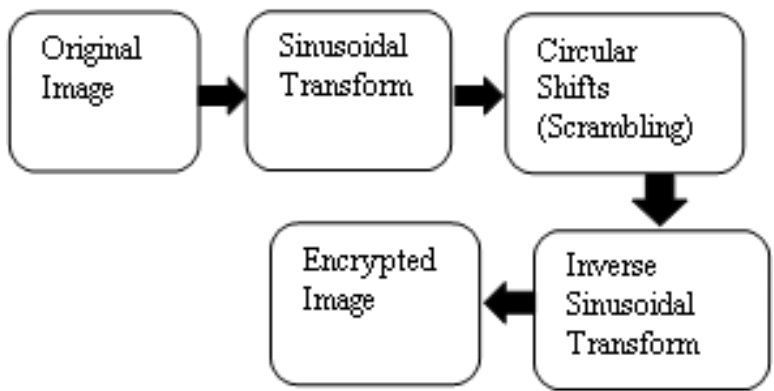

Figure 1: Steps of Encryption

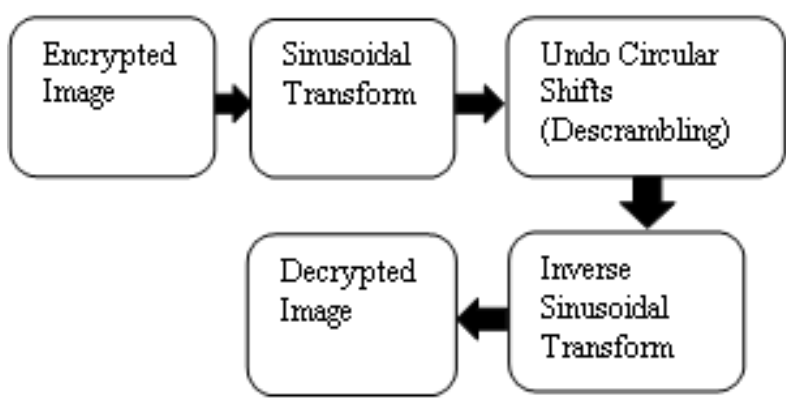

Figure 2: Steps of Decryption

\section{PROPOSED ALGORITHM}

\subsection{Encryption Phase}

1. The image is DCT transformed.

2. Now either perform row-wise or column-wise circular shift, to do that first the image is divided into two parts and circular shifted by a number which can be user input key.

3. Keep dividing the image into halves and repeat step 2.

4. After all circular shifts are done perform inverse DCT transform on the image

5. This completes the encryption stage.

\subsection{Decryption Phase}

1. The image which is received from the encryption phase is again DCT transformed.

2. Divide the image into half and undo the circular shift

3. Divide the two halves into halves and undo the circular shifts

4. Keep repeating step 3 till you get back the image.

5. After the circular shifts are undone apply inverse DCT transform and get back the original image. 


\subsection{DCT Transform}

Discrete Cosine Transform expresses an image into a summation of Cosine functions that converts it into the frequency domain [4]. DCT transform contains the real coefficients and is more efficient than Discrete Sine Transform [4]. The general equation for 2D discrete cosine transform is as follows.

$$
\begin{aligned}
& \mathrm{F}(\mathrm{m}, \mathrm{n})=\frac{2}{\sqrt{\mathrm{MN}}} \mu(\mathrm{m}) \mu(\mathrm{n}) \sum_{x=0}^{M-1} \sum_{y=0}^{N-1} f(x, y) \\
& \cos \frac{(2 x+1) m \pi}{2 \mathrm{M}} \cos \frac{(2 y+1) n \pi}{2 \mathrm{~N}}
\end{aligned}
$$

Where $\mu(m), \mu(n)=\frac{1}{\sqrt{2}}$ for $m, n=0$ and $C(m), C(n)=1$ otherwise.

\subsection{Example}

Step 1: Representing image as a matrix

$\left[\begin{array}{llllllll}12 & 34 & 15 & 76 & 55 & 99 & 14 & 26 \\ 47 & 89 & 111 & 94 & 48 & 58 & 41 & 78 \\ 18 & 87 & 45 & 44 & 89 & 41 & 79 & 22 \\ 85 & 98 & 77 & 49 & 41 & 97 & 99 & 45 \\ 11 & 54 & 165 & 47 & 19 & 22 & 44 & 94 \\ 46 & 41 & 66 & 17 & 25 & 24 & 11 & 41 \\ 18 & 94 & 92 & 44 & 56 & 129 & 41 & 77 \\ 07 & 01 & 98 & 11 & 24 & 26 & 27 & 01\end{array}\right]$

Step 2: Divide the matrix into half.

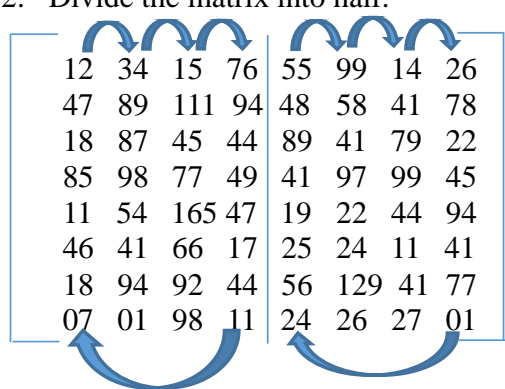

Step 3: Circular- Shift the two halves of the matrix

$\left.\begin{array}{|llll|lccc}76 & 12 & 34 & 15 & 26 & 55 & 99 & 14 \\ 94 & 47 & 89 & 111 & 78 & 48 & 58 & 41 \\ 44 & 18 & 87 & 45 & 22 & 89 & 41 & 79 \\ 49 & 85 & 98 & 77 & 45 & 41 & 97 & 99 \\ 47 & 11 & 54 & 165 & 94 & 19 & 22 & 44 \\ 17 & 46 & 41 & 66 & 41 & 25 & 24 & 11 \\ 44 & 18 & 94 & 92 & 77 & 56 & 129 & 41 \\ 11 & 07 & 01 & 98 & 01 & 24 & 26 & 27\end{array}\right]$

Step 4: Divide the two parts of the matrix into halves.

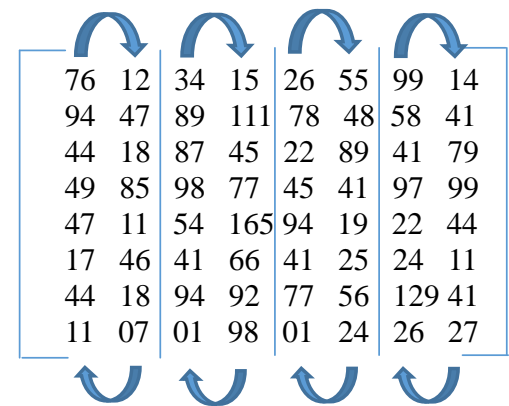

Step 5: Encrypted matrix.

$\left.\begin{array}{rr|ll|ll|ll}12 & 76 & 15 & 34 & 55 & 26 & 14 & 99 \\ 47 & 94 & 111 & 89 & 48 & 78 & 41 & 58 \\ 18 & 44 & 45 & 87 & 89 & 22 & 79 & 41 \\ 85 & 49 & 77 & 98 & 41 & 45 & 99 & 97 \\ 11 & 46 & 165 & 54 & 19 & 94 & 44 & 22 \\ 46 & 17 & 66 & 41 & 25 & 41 & 11 & 24 \\ 18 & 44 & 92 & 94 & 56 & 77 & 41 & 129 \\ 07 & 11 & 98 & 01 & 24 & 01 & 27 & 26\end{array}\right]$

\section{PERFORMANCE MEASURES \\ - Correlation}

An important aspect of similarity measure is image matching. Image matching has many applications some of them being facial recognition, tracking, classification and change detection [5]. Correlation is one of the image matching technique that is widely used. Correlation helps find similarity between two images by comparing the pixel's neighborhood [5]. In this paper correlation helps find the degree of similarity between the original image and restructured image i.e. the one obtained after encryption and decryption.

The Pearson coefficient is one of the ways to measure correlation. It is given by

$$
\mathrm{r}=\frac{n\left(\sum x y\right)-\left(\sum x\right)\left(\sum y\right)}{\sqrt{\left[n \sum x^{2}-\left(\sum x\right)^{2}\right]} \sqrt{\left[n \sum y^{2}-\left(\sum y\right)^{2}\right.}}
$$

Correlation coefficient $r$ lies between $-1 \& 1$. Where $r=-1$ indicates a strong negative relationship between $\mathrm{x} \& \mathrm{y}$ and $\mathrm{r}=$ 1 indicates a strong positive association between $\mathrm{x} \& \mathrm{y} \cdot \mathrm{r}=0$ indicates that $\mathrm{x} \& \mathrm{y}$ are not related at all [6].

\section{- $\quad$ Root Mean Square Error}

The equation of root mean square error is as follows [7]

$e_{r m s}=\sqrt{\frac{\sum_{j=1}^{n}\left(\bar{x}_{j}-x_{j}\right)^{2}}{n}}$

Where $\bar{x}_{j}$ is the $\mathrm{j}^{\text {th }}$ pixel of the restructured image

$$
\begin{aligned}
& x_{j} \text { is the } \mathrm{j}^{\text {th }} \text { pixel of the original image } \\
& \mathrm{n} \text { is the total number of pixels }
\end{aligned}
$$

\section{- Peak Signal-To-Noise Ratio}

PSNR is used for quality detection of the reconstructed image; higher value of PSNR is an indicator of better quality of the reconstructed image [7].

PSNR is given by $10 \log _{10} \frac{R^{2}}{M S E}$

\section{- Entropy}

Entropy of an image is based on different grey levels that image contains.[8] Entropy is higher for an image with more number of grey levels and is lower if the image has less grey levels. If all the pixels are saturated at a particular grey level then the entropy is zero.[9]

Entropy also is a measure of information content so an image with higher entropy value holds more information. Another important characteristic is that scrambling an image doesn't change its entropy. Shannon's entropy [10] is given as

$$
H=-\sum_{i=1}^{n} p_{i} \log _{2} p_{i}
$$




\section{EXPERIMENTAL RESULTS}

The correlation results for row-wise \& column-wise algorithm are given in Table $1 \& 2$ respectively. The correlation between the original image and the decrypted image is 0.99 and suggests that the images are strongly correlated. In the tables each iteration further divides the image into halves and circular shifts those halves. The Entropy of the original image was calculated to be 7.4455 . The Entropy of the original and restructured image is nearly same according to the table data and hence there is negligible information loss

The image $3(\mathrm{a})$ is the original $512 \times 512$ image. $3(\mathrm{~b})$ to $3(\mathrm{~g})$ are row-wise encrypted images and Figure 4(a) to 4(d) are column wise circular shifted in both the cases the number of iterations depict the number of halves the image is divided into. In the $1^{\text {st }}$ iteration an outline of the original image is visible but as the number of iterations increase the obscureness of the image increases

Table 1: The following table shows the Correlation, Root Mean Square Error (RMSE), and Peak Signal to Noise Ratio (PSNR) results corresponding to row circularshifted image.

\begin{tabular}{|c|c|c|c|}
\hline $\begin{array}{c}\text { After } \\
\text { iteration }\end{array}$ & Correlation & RMSE & PSNR \\
\hline 1 & 0.9976 & 3.5497 & 37.1269 \\
\hline 2 & 0.9973 & 3.7360 & 36.6827 \\
\hline 3 & 0.9979 & 3.2871 & 37.7944 \\
\hline 4 & 0.9987 & 2.6819 & 39.5619 \\
\hline 5 & 0.9987 & 2.6977 & 39.5109 \\
\hline 6 & 0.9988 & 2.5491 & 40.0031 \\
\hline 7 & 0.9988 & 2.5633 & 39.9548 \\
\hline 8 & 0.9989 & 2.5367 & 40.0453 \\
\hline 9 & 0.9990 & 2.4223 & 40.4461 \\
\hline 10 & 0.9990 & 2.4223 & 40.4461 \\
\hline
\end{tabular}

Table 2: Entropy of the original, encrypted and restructured image for 10 iterations. The results correspond to row circular-shifted image.

\begin{tabular}{|c|c|c|c|}
\hline $\begin{array}{c}\text { After } \\
\text { iteration }\end{array}$ & $\begin{array}{l}\text { Entropy } \\
\text { of } \\
\text { Original } \\
\text { image }\end{array}$ & $\begin{array}{l}\text { Entropy of } \\
\text { Scrambled } \\
\text { image }\end{array}$ & $\begin{array}{l}\text { Entropy of } \\
\text { Decrypted } \\
\text { image }\end{array}$ \\
\hline 1 & 7.4482 & 7.5393 & 7.4370 \\
\hline 2 & 7.4482 & 7.5445 & 7.4405 \\
\hline 3 & 7.4482 & 7.5563 & 7.4430 \\
\hline 4 & 7.4482 & 7.5692 & 7.4434 \\
\hline 5 & 7.4482 & 7.5685 & 7.4435 \\
\hline 6 & 7.4482 & 7.5715 & 7.4438 \\
\hline 7 & 7.4482 & 7.5715 & 7.4436 \\
\hline 8 & 7.4482 & 7.5719 & 7.4436 \\
\hline 9 & 7.4482 & 7.5735 & 7.4437 \\
\hline 10 & 7.4482 & 7.5735 & 7.4437 \\
\hline & & & \\
\hline
\end{tabular}

Table 3: The following table shows the Correlation, Root Mean Square Error (RMSE), and Peak Signal to Noise Ratio (PSNR) results corresponding to column circularshifted image.

\begin{tabular}{|c|c|l|l|}
\hline $\begin{array}{l}\text { After } \\
\text { iteration }\end{array}$ & Correlation & RMSE & PSNR \\
\hline 1 & 0.9976 & 3.5264 & 37.1842 \\
\hline 2 & 0.9976 & 3.4819 & 37.2944 \\
\hline 3 & 0.9980 & 3.2080 & 38.0060 \\
\hline 4 & 0.9983 & 3.0249 & 38.5166 \\
\hline 5 & 0.9987 & 2.6781 & 39.5744 \\
\hline 6 & 0.9988 & 2.5839 & 39.8853 \\
\hline 7 & 0.9987 & 2.6366 & 39.7099 \\
\hline 8 & 0.9988 & 2.6105 & 39.7964 \\
\hline 9 & 0.9988 & 2.5841 & 39.8848 \\
\hline 10 & 0.9988 & 2.5841 & 39.8848 \\
\hline & & & \\
\hline
\end{tabular}

Table 4: Entropy of the original, encrypted and restructured image for 10 iterations. The results correspond to column circular-shifted image.

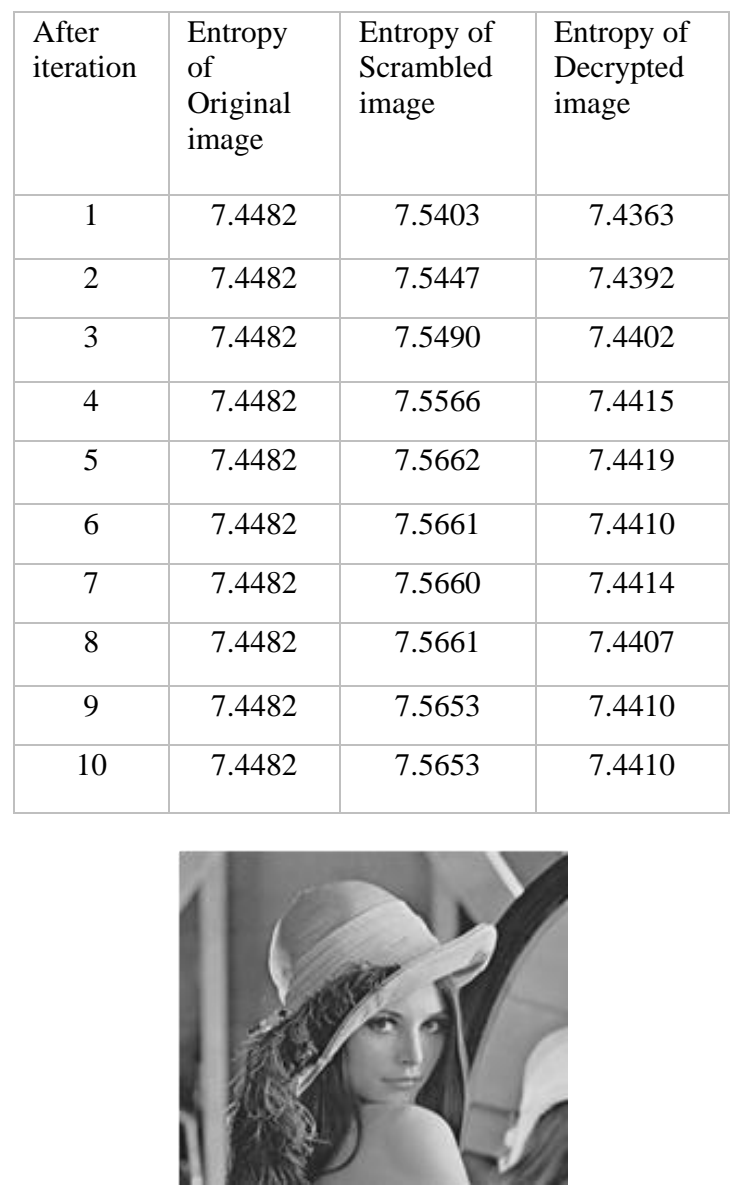

Figure 3(a): Original image 


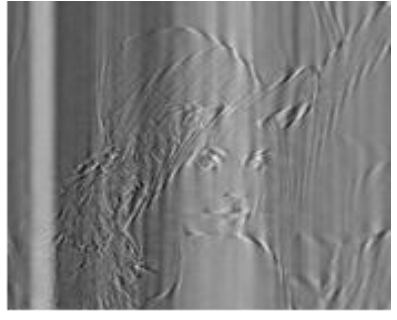

Figure 3(b):Row-wise encryptedimage after 1 iteration.

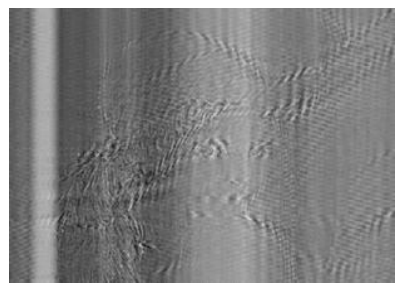

Figure 3(c): Row-wise encryptedimage after 2 iterations.

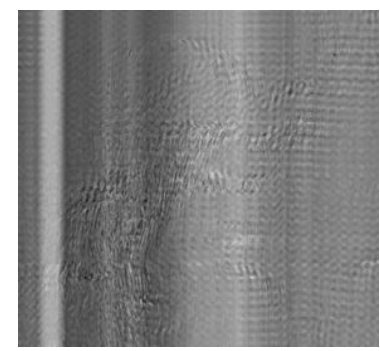

Figure 3(d): Row-wise encryptedimage after 3 iterations.

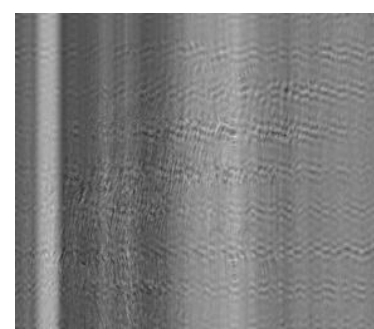

Figure 3(e): Row-wise encrypted image after 4 iterations.

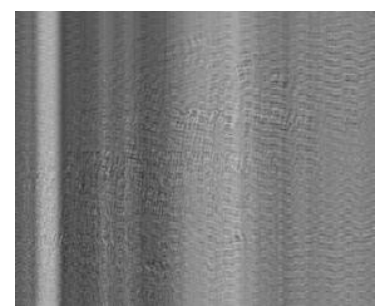

Figure 3(f): Row-wise encrypted Image after 5 iterations

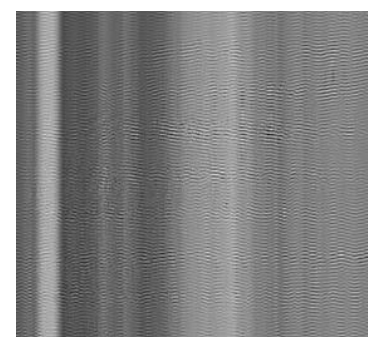

Figure 3(g):Row-wise encrypted

image after 10 iteration.

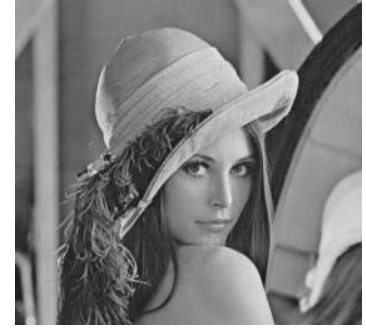

Figure 3(h): Image obtained afterdecryption.

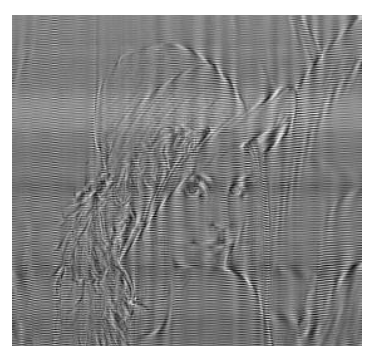

Figure 4(a): Column-wise encrypted image after 1 iterations.

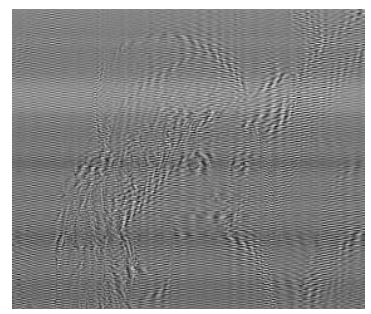

Figure 4(b): Column-wise encryptedimage after 2 iterations.

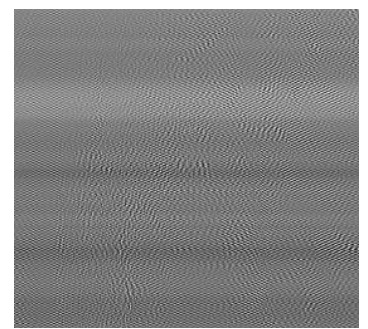

Figure 4(b): Column-wise encrypted Image after 3 iterations.

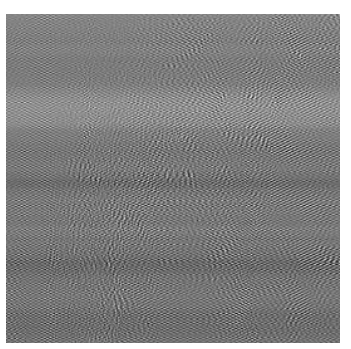

Figure 4(c): Column-wise encrypted image after 5 iterations. 


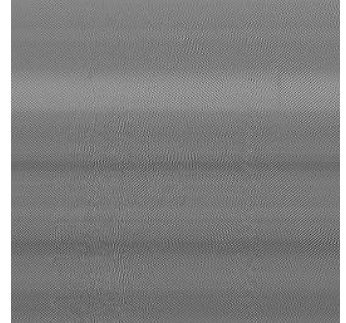

Figure 4(c): Column-wise encrypted image after 10 iterations.

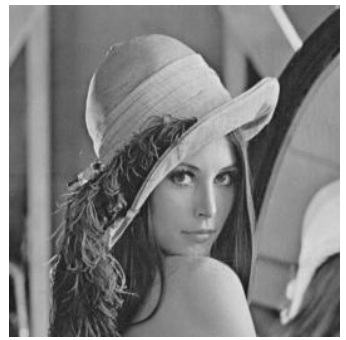

Figure 4(e): Image obtained after Decryption.

\section{CONCLUSION}

Thus the novel approach to image encryption presented by this paper using circular shift is a secure and efficient method for encryption of images. The correlation and entropy values obtained verify that the image obtained after decryption suffers negligible loss and matches the original image to a greater extent. Also applying sinusoidal transform provides security to the algorithm and reduces chances of it being hacked. The algorithm is also simple to use and gives a higher degree of accuracy.

\section{REFERENCES}

[1] John Justin M, Manimurugan S., "A Survey on Various Encryption Techniques", International Journal of Soft Computing and Engineering (IJSCE) ISSN: 2231-2307, Volume-2, Issue-1, March 2012.

[2] Rinki Pakshwar, Vijay K. Trivedi, Vineet Richhariya, “A Survey on Different Image Encryption and Decryption Techniques", International Journal of Computer Science and Information Technologies (IJCSIT), Vol. 4 (1), 2013, $113-116$
[3] Nicholas J. Daras, Michael Th. Rassias Computation, Cryptography, and Network Security Springer, 16-Sep2015

[4] H. B. Kekre, Tanuja Sarode, Pallavi N. Halarnkar \& Debkanya Mazumder, "Comparative Performance of Image Scrambling in Transform Domain using Sinusoidal Transforms", International Journal of Image Processing (IJIP), Vol. 9, issue 2, 2014.

[5] Tai-hoon Kim, Hojjat Adeli, Carlos Ramos, Byeong-Ho Kang Springer, "Signal Processing, Image Processing and Pattern Recognition: International Conferences, SIP 2011, Held as Part of the Future Generation Information Technology Conference, FGIT 2011, in Conjunction with GDC 2011, Jeju Island, Korea, December 8-10, 2011. Proceedings", 02-Dec-2011

[6] Mintu Philip, "An Enhanced Chaotic Image Encryption", International Journal of Computer Science, Engineering and Information Technology (IJCSEIT), Vol.1, No.5, Dec 2011

[7] Hala Bahjat, May A.Salih, "Dynamic Shuffling for Speed Image Encryption", International Journal of Computer Applications (0975-8887), Vol. 89, No. 7, March 2014

[8] Sowmyashree, R.R. Sedamkar, "A Hybrid Approach for Secure Data Communication using Reversible Data Hiding and Image Encryption", International Journal of Current Engineering and Technology, Vol. 4, No. 6, Dec 2014

[9] Dr. H.B. Kekre, Sudeep D. Thepade, Tanuja K. Sarode, Vashali Suryawanshi, "Image Retrieval using Texture Features extracted from GLCM, LBG, KPE", International Journal of Computer Theory and Engineering, Vol. 2, No. 5, October 2010, 1793-8201.

[10] Yue Wu, Yicong Zhou, George Saveriades, Sos Agaian, Joseph P. Noonan, Premkumar Natrajan, "Local Shannon entropy measure with statistical tests for image randomness", Information Sciences, Vol. 222, February 10, 2013, Pages 323-342. 